\title{
GENERALIZATION OF DUAL STRUCTURAL OPTIMIZATION PROBLEMS IN TERMS OF FRACTIONAL PROGRAMMING*
}

BY

A. J. MORRIS

Structures Department, Royal Aircraft Establishment, Farnborough

Summary. Duality now plays an important role in the theory of optimum structures but has not been given adequate detailed consideration within this context. The paper makes a limited attempt to satisfy this requirement through a generalization of the associated duality theory by formulating the structural optimization as a fractional program. This provides some new forms for the dual objective function and crystalizes some of the intrinsic problems associated with dual structural programs.

1. Introduction. Duality theory has played an important role in the development of structural optimization theory and the associated computer-based solution algorithms. Normally the problems examined have centered on the minimum-weight design of structures subject to a variety of behavioral constraints which are of practical importance in the fabrication of aerospace structures.

Early applications of the theory [1] show that certain formulations may be conveniently described in terms of the standard duality theory of linear programming. Unfortunately, many problems do not fit into this category and recent efforts have been devoted to the use of the more complex nonlinear duality theory. An early and somewhat straightforward application was to check the validity of the popular stress-ratioing method and to providing a corrective strategy when the method converges towards non-optimal solutions [2]. Recently Templeman [3] has attempted a more comprehensive exploitation of the dual problem to provide a rapidly convergent algorithm for the optimum design of general pinjointed frameworks.

In view of these efforts it is appropriate to attempt a generalization of the theory, and the present paper is concerned with employing duality in fractional programming to generalize some aspects of structural optimization duality theory. In particular we seek to generalize a dual form proposed by Bartholomew [4], originally for frameworks, which gives a closer bound than the usual formulation on local optima. Since many structural optimization problems can be described in terms of homogeneous polynomials when cast in fractional form, this formulation is adhered to in the sequel. Thus, we examine the duality theory of fractional programming where the various functions are assumed to be positively homogeneous polynomials. The paper in some ways constitutes a special case of the known theory of duality in fractional programming [5-9]. Although the functions employed in the next section are described in terms of polynomials, after the problem

\footnotetext{
* Received November 22, 1977; revised version received January 26, 1978. Copyright $\odot$ Controller HMSO London 1978.
} 
formulation the initial discussions relate to general fractional programs; only subsequently is the argument entirely particularized to polynomials forms.

2. Duality theory. Although optimized designs can be sought for a wide range of structural configurations, the chief characteristics associated with this class of problem appear in the simple case of the minimum-weight design of pin-jointed frameworks. Taking the specific case of a stress- and displacement-constrained structure, a typical problem is to find a vector of bar cross-sectional areas $x^{*}=\left(x_{1}{ }^{*}, x_{2}{ }^{*}, \cdots, x_{r}{ }^{*}\right)^{t}$ which minimize the weight

$$
W(x)=\sum_{i=1}^{r} \rho_{i} l_{i} x_{i}
$$

subject to displacement constraints

$$
\bar{\Delta}_{j} \geq \Delta_{j}=\sum_{i=1}^{r} \frac{S_{i}(x) l_{i} s_{l j}(x)}{E_{i} x_{i}} \quad(j=1,2, \cdots, k)
$$

and stress constraints

$$
\bar{\sigma}_{i} \geq\left|S_{i}(x) / x_{i}\right| \quad(i=1,2, \cdots r)
$$

where $\Delta_{j}$ represents a nodal displacement; $S_{i}$ is the force in the $i$ th bar due to the applied loads; $s_{i j}$ is the force in bar $i$ due to the application of a unit load at the $j$ th node; $l_{i}, \rho_{i}$ are the length and weight/unit volume respectively and $E_{i}$ denotes Young's modulus. In the case of statistically determinate structures the bar forces are independent of the crosssectional areas and are functions of the applied loads only. For this structure the problem has a linear objective function with nonlinear polynomial constraints. When statically indeterminate structures are considered the situation is more complex as the bar forces are no longer independent of bar areas, but the basic mathematical structure of the constraints is preserved. An alternative formulation suggested by convexity requirements involves new design variables which are the inverse of bar cross-sectional areas, i.e. $z_{i}=1 / x_{t}(i=1$, $2, \cdots r)$. Problems involving statically determinate structures are now described by nonlinear objective function subject to linear constraints. Comparable changes occur when the transformation is applied to the statically indeterminate case.

For more complex situations the finite-element method would be employed in calculating the structural responses under the action of the design loads. The finite-element method permits the analysis of complex structures and the imposition of a wide range of constraints which together reflect a more realistic spectrum of design cases. For more complex structures the simple objective functions appropriate to frameworks can be replaced by higher-order functions which in some cases may be empirically derived. Nevertheless, for many design situations the basic mathematical structure given above is preserved and thereby allows the problem to be formulated as a fractional programming problem described in terms of polynomial functions. An additional pleasing feature, indicated by the framework example, is that these polynomials are often positively homogeneous.

On this basis a useful general form for the structural optimization problem is given by considering both the objective function and the constraints to be described in terms of fractional functions. Hence we are seeking a vector of design variables $x^{*}=\left(x_{1}, x_{2}, \cdots\right.$, $\left.x_{r}\right)^{t}$ which minimize

$$
W(x)=f(x) / h(x)
$$


subject to

$$
u_{\jmath} \geq g_{\jmath}(x) / l_{\jmath}(x)
$$

where $f(x)$ is a homogeneous polynomial of order $n, h(x)$ is a homogeneous polynomial of order $q, g_{j}(x)$ are homogeneous polynomials of order $m(j=1, \cdots t), l_{j}(x)$ are homogeneous polynomials of order $p(j=1, \cdots t)$, and

$$
h(x)>0, \quad l_{j}(x)>0 \quad(j=1, \cdots t) .
$$

The Lagrangian function associated with this minimization problem is given by

$$
L(x, \lambda)=W(x)+\sum_{j=1}^{t} \lambda_{j}\left(\frac{g_{j}(x)}{l_{j}(x)}-u_{j}\right)
$$

and normally one hopes that this function is convex. Unfortunately, with (2.1), unless there is an exceptional form for the function $h(x)$, the Lagrangian is, at best, pseudoconvex in the design variables, i.e.

$$
\nabla_{x} L(x, \lambda)\left(x^{*}-x\right) \geq 0 \Rightarrow L\left(x^{*}, \lambda\right) \geq L(x, \lambda) .
$$

As may be easily demonstrated, the pseudo-convexity property requires that the functions $f(x), g_{j}(x)$ be convex and $h(x), l_{j}(x)$ concave. Thus, in order to complete the definition of the optimization problem (2.1) additional statements are required concerning the design variables and functions:

(a) $x^{*} \in X^{o}$ where $X^{o} C R^{r}$ is an open convex set;

(b) $f(x): X^{o} \rightarrow R$ is convex and differentiable on $X^{o}$;

(c) $g_{j}(x): X^{o} \rightarrow R^{t}$ are convex and differentiable on $X^{o}$;

(d) $h(x): X^{o} \rightarrow R$ is concave and differentiable on $X^{o}$;

(e) $l_{j}(x): X^{o} \rightarrow R^{t}$ are concave and differentiable on $X^{o}$.

For this problem the Kuhn-Tucker sufficient optimality conditions [5] require that

$$
\begin{aligned}
& \nabla\left(\frac{f\left(x^{*}\right)}{h\left(x^{*}\right)}\right)+\sum_{j=1}^{t} \lambda_{\jmath}^{*} \nabla\left(\frac{g_{\jmath}\left(x^{*}\right)}{l_{\jmath}\left(x^{*}\right)}-u_{\jmath}\right)=0, \quad\left(\frac{g_{\jmath}\left(x^{*}\right)}{l_{\jmath}\left(x^{*}\right)}-u_{\jmath}\right) \leq 0, \\
& \lambda_{\jmath} *\left(\frac{g_{\jmath}\left(x^{*}\right)}{l_{\jmath}\left(x^{*}\right)}-u_{\jmath}\right)=0, \quad \lambda_{\jmath}^{*} \geq 0, x^{*} \in X^{o}, \quad j=(1,2, \cdots t) .
\end{aligned}
$$

Using these conditions, we can now proceed to derive a suitable duality theory and, to aid progress, the set $W$ is introduced, where

$$
W=\left\{(x, \lambda) \mid x \in X^{o}, \quad \lambda \in R^{t}, \quad \nabla_{x} L(x, \lambda)=0\right\} .
$$

Following Bector [7], a weak duality theorem can now be defined.

THEOREM: Let $x \in X^{\circ}$ and $\left(x^{*}, \lambda^{*}\right) \in W$ and satisfy the Kuhn-Tucker conditions; then $f\left(x^{*}\right) / h\left(x^{*}\right) \geq L\left(x^{*}, \lambda^{*}\right)$.

Proof: Set $\phi_{0}(x)=f(x) / h(x), \phi_{J}(x)=\left(g_{\jmath}(x) / l_{j}(x)\right)-u_{j},(j=1, \cdots t)$ and hence the functions $\phi_{l}(x)$ are pseudoconvex. Thus

$$
\phi_{0}(x) \geq \phi_{0}\left(x^{*}\right)-\nabla \phi_{0}\left(x^{*}\right)\left(x-x^{*}\right)
$$

because $\left(x^{*}, \lambda^{*}\right) \in W$. Then

$$
\nabla \phi_{0}\left(x^{*}\right)=-\sum_{j=1}^{t} \lambda_{j}^{*} \nabla \phi_{j}\left(x^{*}\right) .
$$


Hence

$$
\phi_{0}(x) \geq \phi_{0}\left(x^{*}\right)+\sum_{j=1}^{t} \lambda_{j}^{*} \nabla \phi_{j}\left(x^{*}\right)\left(x-x^{*}\right) .
$$

The Lagrangian function is pseudoconvex and this implies $\lambda_{j}^{*} \phi_{j}(x) \geq \lambda_{f}^{*} \phi_{j}\left(x^{*}\right)$; thus

$$
\begin{aligned}
& \phi_{0}(x) \geq \phi_{0}\left(x^{*}\right)+\sum_{j=1}^{t} \lambda_{j}^{*}\left(\phi_{j}\left(x^{*}\right)-\phi_{j}(x)\right) \\
& \geq L\left(x^{*}, \lambda^{*}\right)-\sum_{j=1}^{m} \lambda_{j}^{*} \phi_{j}(x) \geq L\left(x^{*}, \lambda^{*}\right)
\end{aligned}
$$

or

$$
f(x) / h(x) \geq L\left(x^{*}, \lambda^{*}\right) .
$$

Based on this theorem a suitable dual problem is obtained by requiring the maximization of the Lagrangian function for $(x, \lambda) \in W$.

Differentiating $L(x, \lambda)$ with respect to the design variables for $(x, \lambda) \in W$ and using Euler's theorem gives

$$
\bar{x} \nabla_{x} L(x, \lambda)=\frac{n f(x)}{h(x)}-\frac{q f(x)}{h(x)}+\sum_{j=1}^{t} \lambda_{j} \frac{g_{j}(x)}{l_{j}(x)}(m-p)=0
$$

or

$$
(n-q) \frac{f(x)}{h(x)}+(m-p) \sum_{j=1}^{t} \lambda_{j} \frac{g_{j}(x)}{l_{j}(x)}=0 .
$$

Substituting this equation into the Lagrangian function generates a dual objective function of the form

$$
D(x, \lambda)=\frac{f(x)}{h(x)}-\frac{(n-q)}{(m-p)} \frac{f(x)}{h(x)}-\sum_{j=1}^{t} \lambda_{j} u_{j}
$$

or

$$
D(x, \lambda)=\frac{(m-p-n+q)}{(m-p)} \frac{f(x)}{h(x)}-\sum_{j=1}^{t} \lambda_{j} u_{j}
$$

Thus the dual problem is to maximize (2.3) subject to Eqs. (2.2) and the positivity of the Lagrangian multipliers $\lambda_{j}(j=1, \cdots t)$.

An alternative form for the objective function (2.3) is found by noting [4] that a linear structure can always be scaled. That is, a specific vector of design variables $x$ can be uniformly scaled to produce a new design vector $s x$. Thus we have the scale factor as a free variable in the dual objective (2.3) which can be maximized with respect to this factor. Putting the scale factor explicitly into (2.3) gives

$$
\frac{(m-p-n+q)}{(m-p)} s^{(n-q)} \frac{f(x)}{h(x)}-F s^{(p+n-m-q)}
$$

where $F=\sum_{j=1}^{t} \lambda_{j} u_{j}$. Differentiating in terms of $s$ and solving yields

$$
s^{(p-m)}=\frac{(n-q)}{(p-m)} \frac{f(x)}{h(x) F}
$$


which can then be used to derive the alternative dual objective function of the form

$$
\frac{f(x)}{h(x)}\left\{\frac{(n-q)}{(p-m)} \frac{f(x)}{h(x) F}\right\}^{(n-q) /(p-m)} .
$$

Whilst Bartholomew has shown [4] that (2.4) can provide a closer bound on the local optimum in the minimum weight design of frameworks, we note that it is less generally applicable than (2.3).

Taking some simple specific examples, the stress-constrained framework studied by Hemp [1] requires that $h(x), l_{j}(x)$ are deleted and that $n=m=1$, whereupon (2.3) and (2.4) provide the same dual objective function $-F$. For a displacement-constrained framework using cross-sectional areas as the design variables $q=0, p=2, m=1$ and (2.4) gives

$$
f(x)\left\{\frac{n f(x)}{F}\right\}^{n}
$$

whilst (2.3) provides the form

$$
(1+n) f(x)-F \text {. }
$$

For minimum-volume designs with a fixed layout, $n=1$ and the above reduce to the expressions given by Bartholomew [4].

3. Conclusions. Although the previous section has generalized some aspects of the duality theory of structural optimization, it also highlights some of the intrinsic problems. In particular, the requirements of convexity and concavity on the constitutive functions are particularly severe. Many of the problems encountered in structural design do not satisfy these conditions and the use of duality theory in these cases is of limited value. Nevertheless, at points close to a local optimum the theory is still valid and valuable as a terminating criterion in structural optimization algorithms.

\section{REFERENCES}

[1] W. S. Hemp, Optimum structures, Oxford, 1973

[2] P. Bartholomew and A. J. Morris, A unified approach to fully-stressed design, Eng. Optimisation 2, 3-15 (1976)

[3] A. B. Templeman, A dual approach to optimum stress design, J. Struct. Mech. 4, 235-257 (1976)

[4] P. Bartholomew, A dual bound used for monitoring structural optimisation programs, Eng. Optimization, to appear

[5] Olvi Mangasarian, Non-linear programming, McGraw-Hill, 1969

[6] R. Jagannathan, Duality in non-linear fractional programming, Z. Operations Research 17, 1-3 (1973)

[7] C. R. Bector, Duality in non-linear programming, Z. Operations Research 17, 183-193 (1973)

[8] S. Schaible, Parameter-free convex equivalent and dual programs of fractional programming problems, $Z$. Operations Research 18, 187-196 (1974)

[9] D. G. Mahajan and M. N. Vartak, Generalisation of some duality theorems in non-linear programming, Mathematical Programming 12, 293-317 (1977) 